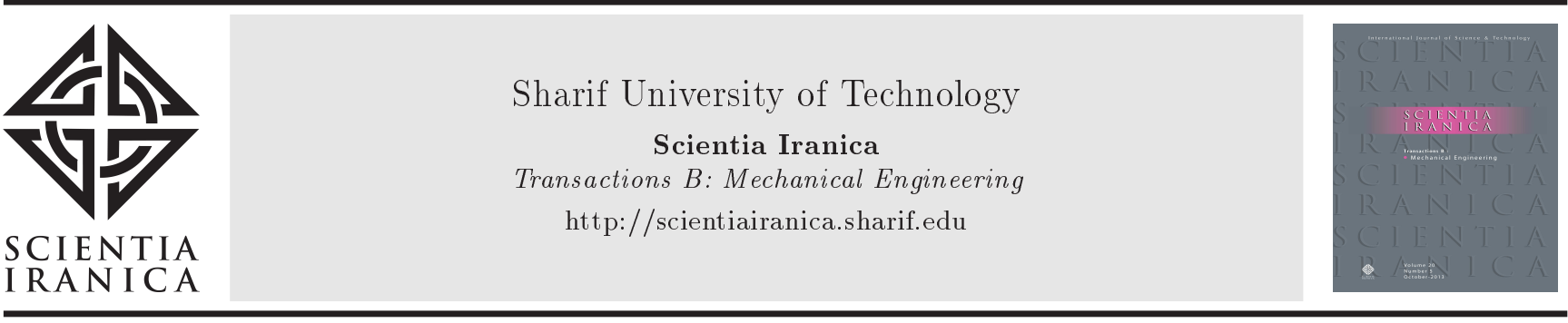

\title{
Modeling particle deposition in the respiratory system during successive respiratory cycles
}

\author{
H. Nemati, M.S. Saidi*, and V. Hosseini \\ Department of Mechanical Engineering, Sharif University of Technology, Tehran, P.O. Box 11155-9567, Iran.
}

Received 19 April 2017; received in revised form 16 April 2018; accepted 16 February 2019

\section{KEYWORDS}

Particle deposition; Respiratory system; Lung;

Lobe;

Successive respiratory cycles.

\begin{abstract}
This study uses a 5-lobe symmetric model to investigate total, lobar and generational particle deposition fractions in the lungs during successive cycles. It was found that for the particle size between 0.05 and $2 \mu \mathrm{m}$ and the tidal volumes greater than $1000 \mathrm{ml}$, the effect of successive cycles helped predict more deposition fraction per cycle up to about $16 \%$ than that of a single cycle. The mentioned range of tidal volumes corresponds to light or heavy physical activities. Therefore, it can be understood that people, when physically active, exposed to particulate matter within the mentioned size range are at higher health risk as compared to both the resting state and the same state calculations based on a single cycle. Finally, total and generational remaining mass fractions suspended in the respiratory tract after the completion of each cycle are calculated. This remaining mass fraction turned out to be negligible except for particles between 0.05 and $2 \mu \mathrm{m}$.
\end{abstract}

(C) 2020 Sharif University of Technology. All rights reserved.

\section{Introduction}

The investigation of the amount and pattern of particle deposition in the human respiratory system has drawn many researchers' attention during recent years. These studies are carried out with the objective of achieving two main goals: The first one is to study and control the adverse effects of particulate matter air pollution on human health; the second one is to investigate and increase the deposition rate of aerosolized drug particles in the respiratory system. To treat some diseases, specifically pulmonary diseases, aerosolized drugs are prepared and inhaled. If the particles released from such equipment are in the proper amount and reach proper spots, the treatment can occur effectively.

\footnotetext{
*. Corresponding author. Tel.: +982166165558 E-mail addresses: nemati52@hotmail.com (H. Nemati); mssaidi@sharif.edu (M.S. Saidi); vhosseini@sharif.edu (V. Hosseini)
}

doi: $10.24200 /$ sci. 2019.21269
So far, a lot of modeling has been done in the field of particle deposition in the respiratory system. Some researchers have studied this problem locally, e.g., in the oral or nasal cavity. On the other hand, some others have developed the whole lung models or models that represent the whole respiratory system. One of the first such studies was done by Yeh and Shcum [1]. They calculated the particle deposition in a single path of the respiratory tract. In addition to introducing a novel statistical model in their research series, Koblinger and Hofmann [2-6] simulated the particle deposition in the respiratory tract using empirical formulas according to Yeh and Schum [1]. Dastanpour et al. [7] modeled the whole lung particle deposition based on the statistical model using a Monte-Carlo method. They presented a novel technique to calculate the airflow in each airway based on the position of the airway and the alveoli distal to it. Monjezi et al. [8] used a multi-scale method to develop the whole lung particle deposition model. They coupled a 3D model of the upper airways with an impedance model of the distal airways to calculate the flow in each airway. They also used a statistical 
model to build up the geometric structure of the lungs. Moreover, recently, Georgakakou et al. [9] developed a simplified model based on perfect alveolar mixing. The results they derived for the pulmonary deposition were in good agreement with previous data for particles greater than $0.1 \mu \mathrm{m}$. Asgharian and Price [10] used a multiple-path geometry based on the data presented by Raabe et al. [11] and Yeh and Schum [1] and calculated the particle deposition using the concentration equation. Salma et al. [12] calculated the particle deposition under different physical activity conditions for different regions of Budapest using a statistical geometric model. Their results showed that, for the harder physical activities, the acinar deposition increased, while the tracheobronchial deposition fraction remained almost constant.

In the present study, the effect of successive respiratory cycles on the particle deposition is investigated as a more exact solution to the problem instead of a single cycle that has been studied widely so far. Total and generational deposition fractions and the remaining mass fraction suspended in the respiratory tract after successive cycles until the quasi-steady state of breathing are also calculated and discussed.

\section{Methodology}

In order to simulate the particle deposition in the respiratory system, three major steps must be taken. The first step is the simulation of the geometry of the respiratory tract. Based on the challenge of each problem, the proper geometrical model among different presented geometrical models should be chosen, and one should build up the geometry of the respiratory tract. The second step is the calculation of the airflow in each of the airways. It can be done by using proper hydrodynamic boundary conditions. Finally, the third step is the calculation of the particle deposition fraction in each airway based on the presented deposition formulas. Each of these major steps in this study will be explained in the following sections.

\subsection{Geometry}

In the present study, Yeh and Shum's 5-lobe geometric model [1] is used. In this model, the airways proximal to the starting point of the five lobes are modeled as the asymmetric channels, each of which has its own geometric data. On the other hand, within each of the five lobes, the bifurcations are considered to be symmetric, and the airways in the same generation have the same geometric data. Although the respiratory tract is supposed to be symmetric in each lobe, each of the five lobes has its own geometric data sheet.

Therefore, we can model each lobe separately and capture its own particle deposition pattern. In this model, for each lobe, the generation after which the airways are in the alveolar region is determined. Based on a research by Weibel et al. [13], for the first three generations of the alveolar region, it is supposed that $0.2,0.4$, and 0.7 of the lateral area of the airways is covered by the alveoli, respectively. For the next generations, this area fraction increases to unity. It is implied that, for the generations distal to the third alveolar generation, the lateral area is totally covered by the alveoli. The FRC, which stands for "Functional Residual Capacity", is considered equal to $2300 \mathrm{ml}$ based on physiologic data. Following the application of the mentioned modifications to the model according to Weibel et al. [13], the total number of alveoli is calculated to be about 450 million. Ochs et al. [14] studied the lung of six adults and presented 480 million alveoli, on average, for an adult. This shows that the implemented modification to the original geometric model that presented 300 million alveoli in number could make it closer to the real structure of the lung and, thus, make it more accurate. Although the statistical model, among the geometric models, can predict the uncertainties better than the other models, the current study used a 5-lobe symmetric model. Considering that the challenge of the present study is the effect of successive respiratory cycles, the application of a statistical model during successive cycles can lead to an enormous computational cost, which is out of our computational scope.

\subsection{Flow calculation}

In order to simulate the airflow in the airways, at first, we need to know the temporal breathing profile. In the primary research studies, as the first approximation, the inhalation and exhalation flows were assumed to be constant with respect to time. Thereafter, based on the suggestion of other researchers $[15,16]$, the sinusoidal profile was used frequently. This profile is more similar to the real breathing flow profile and, nevertheless, enjoys simplicity for the calculation algorithm to be run with reasonable time and energy cost.

The other challenge in the simulation of the airflow is the flow division between the airways. Considering the fact that the main reason for the flow creation is the expansion and contraction of the alveoli, this study calculated the flow share of each airway based on the number of the alveoli distal to it. This technique was also used by Henry and Tsuda [17] in 2010 and reused by Dastanpour et al. [7] in 2014. In the alveolar airways, a fraction of the lateral area is covered by the alveoli; therefore, a portion of flow goes into these alveoli and, in accordance, the output flow is somewhat less than the input flow.

In this study, based on the physiological data, the inhalation and exhalation time is considered to be $2 \mathrm{~s}$, and the respiratory pause time (the time between the end of an exhalation and the start of the next 


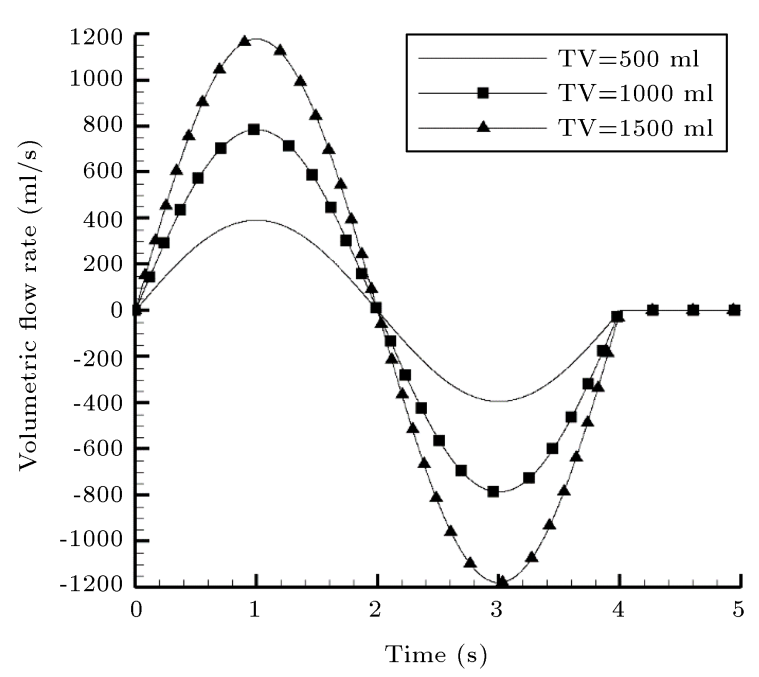

Figure 1. Volumetric flow rate versus time.

inhalation) is considered to be $1 \mathrm{~s}$. The calculation is done for three different values of the tidal volume. A tidal volume of $500 \mathrm{ml}$ is considered to simulate breathing in the normal mode, $1000 \mathrm{ml}$ for the state of light physical activity, and $1500 \mathrm{ml}$ for the state of heavy physical activities. The total air flow as a function of time is depicted in Figure 1 for three different values of the tidal volume.

\subsection{The calculation algorithm}

In this study, it is supposed that a subject is continuously exposed to the particles. It is implied that the boundary condition at the inlet of the respiratory system is the constant concentration of particles during the inhalation and the escape boundary condition for the exited particles during the exhalation. This state can be considered in the case of air pollution calculation. In this problem, two types of numerical integrations are required. The first one is integration on the mass of the particles entered into the oral or nasal cavity, and the second one is to track every group of particles in the respiratory tract. Each of these integrations has its own time step. For both of these numerical integrations, the time-step size study was done. For the integration on the mass of the entered particles, three different time steps of $0.05,0.02$, and $0.01 \mathrm{~s}$ were used and the results were close and in agreement. Thus, the problem was solved based on $0.05 \mathrm{~s}$. For the integration to track every group of particles in the respiratory tract, a time-step between $0.001 \mathrm{~s}$ and $0.0001 \mathrm{~s}$ was chosen based on the geometry of each lobe. Thus, each lobe has its own time-step. For each of the five lobes, the time-step decreased until the results changed negligibly after dividing the timestep by 2 and 4 . Then, this time-step was considered for the corresponding lobe.

The general algorithm to solve the problem has the following four main steps:
1. At the beginning, it is supposed that the whole respiratory system is empty of particles. At this time, breathing begins in the air containing particles. Unity is attributed to the total particle mass entering the respiratory system during the inhalation;

2. In each time step, with a proper understanding of the airflow versus time, the mass fraction entering the respiratory system can be tracked in the respiratory tract, and its deposition fraction in each airway is calculated. After exiting each airway, the remaining particles are divided into two parts and enter the distal airways based on the flow division between the daughter airways. This procedure continues until the first inhalation ends;

3. At the end of the first inhalation, part of the total particle mass is deposited in the airways, part of it enters the alveoli, and the rest is suspended in the airways. The mass entering the alveoli is partly deposited, and the rest is suspended in the alveoli. In the initial state before the inhalation begins, the alveoli contain a volume of air inside them. When the new particles containing air are sucked into the alveoli, this "polluted" air is partly mixed with the "clean" air that exists initially in the alveoli. This amount of mixture is introduced by "mixing factor". Koblinger and Hofmann [2] suggested the amount of 0.25 for this parameter based on best fitting with the experimental results. In this study, this amount is considered for the mixing factor. During the exhalation, part of the suspended mass in the alveoli exits the respiratory system. However, the part that is determined by the mixing factor is mixed with the initial air in the alveoli and exits the alveoli gradually and with a delay to the main wave of the exhalation air. It is implied that the moment when the unmixed part of air exits the alveoli, the exiting process of the mixed part of air begins and lasts until the exhalation ends. This time delay is the reason why, at the end of the exhalation, the particles in the mixed part of the air cannot totally exit the respiratory system and part of it remains in the airways. This is the main mechanism that is responsible for the remaining mass in the respiratory tract at the end of the respiratory cycle. By integrating the time-steps of the first respiratory cycle, the deposited fraction and the remaining fraction of particles mass can be calculated after the first exhalation ends. During the pause time, part of the remaining mass is deposited and the remaining mass decreases slightly.

At this moment, the modeling of the first respiratory cycle is completed, and the deposition fraction and pattern, the remaining mass fraction 
and pattern, and the exited mass fraction after the first respiratory cycle can be obtained;

4. When the second respiratory cycle begins, at first, the location of the remaining mass fractions is tracked, and their own deposition and the remaining mass fraction are calculated. Then, the calculation of the newly entered mass from the oral or nasal cavity is done similar to the first cycle algorithm. The computational algorithm for the next cycles is exactly like the second cycle.

Finally, after a few respiratory cycles, the deposited fraction during each cycle and the remaining mass fraction in the respiratory tract will be accessible. The above computational algorithm was run by a computational code, and the results were extracted. The flowcharts of the particle deposition algorithm in the tracheobronchial and alveolar regions in a typical lobe are presented in Appendix A.

For more clarification, the mass balance equation is presented below. For every stage of the geometry, namely a single alveolus, an airway, and the whole respiratory system, the mass is balanced based on Eq. (1):

$$
m_{\text {rem }}^{\text {old }}+m_{e n}=m_{d e p}+m_{\text {out }}+m_{\text {rem }}^{\text {new }} .
$$

In the above equation, $m_{\text {rem }}^{\text {old }}$ and $m_{\text {rem }}^{\text {new }}$ are the remaining mass fractions at the beginning and end of a typical respiratory cycle, $m_{e n}$ is the entered mass fraction, $m_{\text {out }}$ is the exited mass fraction, and $m_{\text {dep }}$ is the deposited mass fraction during the current cycle in each airway or alveolus. Since the aim of this study is to assess the effect of successive cycles that is basically due to the alveolar mixing, the mass balance equations in a single alveolus are presented below, specifically.

$$
\begin{aligned}
& m_{\text {en }}=m_{m}+m_{u}=m_{e n} * m f+m_{e n} *(1-m f), \\
& m_{\text {dep }}=\left(m_{m}+m_{u}+m_{\text {rem }}^{\text {old }}\right) * d f \\
& m_{\text {out }}=m_{u} *(1-d f)+m_{m} *\left(1-\frac{v_{\text {min }}}{v_{\text {en }}}\right) *(1-d f), \\
& m_{\text {rem }}^{\text {new }}=m_{\text {rem }}^{\text {old }} *(1-d f)+m_{m} * \frac{v_{\text {min }}}{v_{\text {en }}} *(1-d f)
\end{aligned}
$$

In Eqs. (2a) to (2d), $m f$ and $d f$ are the mixing factor and deposition fraction, respectively. Deposition fraction in each airway or alveolus is calculated based on formulas that are to be introduced in the following sections. $m_{m}$ and $m_{u}$ are the mixed and unmixed parts of the entered mass to the alveoli, respectively. In addition, in these equations, $v_{\min }$ and $v_{e n}$ are the minimum volume and the volume of a typical alveolus at a moment when the particle-containing air front enters the alveolus, respectively. The total minimum volume of alveoli calculated using $F R C$ and the volume of the respiratory dead space that are considered to be $2300 \mathrm{ml}$ and $150 \mathrm{ml}$, respectively, in this study. The minimum volume of each alveolus is calculated by dividing the total minimum volume of alveoli by their number (450 million) that gives the amount of $4.78 \mathrm{e}-$ $12 \mathrm{~m}^{3} . v_{e n}$ is different for each alveolus, and its amount is calculated computationally by tracking the particlecontaining air front. Figure 2 depicts a schematic view of the deflection of a typical alveolus and the entrance of the air front into it during a cycle. It may give a good conceptual sense of $v_{e n}$. In Figure 2, the areas with a higher concentration of particles are shown to be darker. This spectrum has no quantitative basis and is depicted merely to clarify the computational algorithm for the reader. Figure 2 shows that the alveolar mixing increases the remaining mass fraction, which still exists from the previous cycle. When the respiration reaches a quasi-steady state, this increment decays and the remaining mass fraction experiences periodic variations along the respiratory cycles.

In the present study, deposition due to three major deposition mechanisms, e.g., impaction, sedimentation, and diffusion, is calculated. In the oral and nasal cavities, to the best of our knowledge, no research has been done to estimate the deposition fraction purely by sedimentation. Moreover, due to the small residence time of the particles in these cavities, the deposition by sedimentation can be neglected. Therefore, for the oral and nasal cavities, the deposition is calculated based
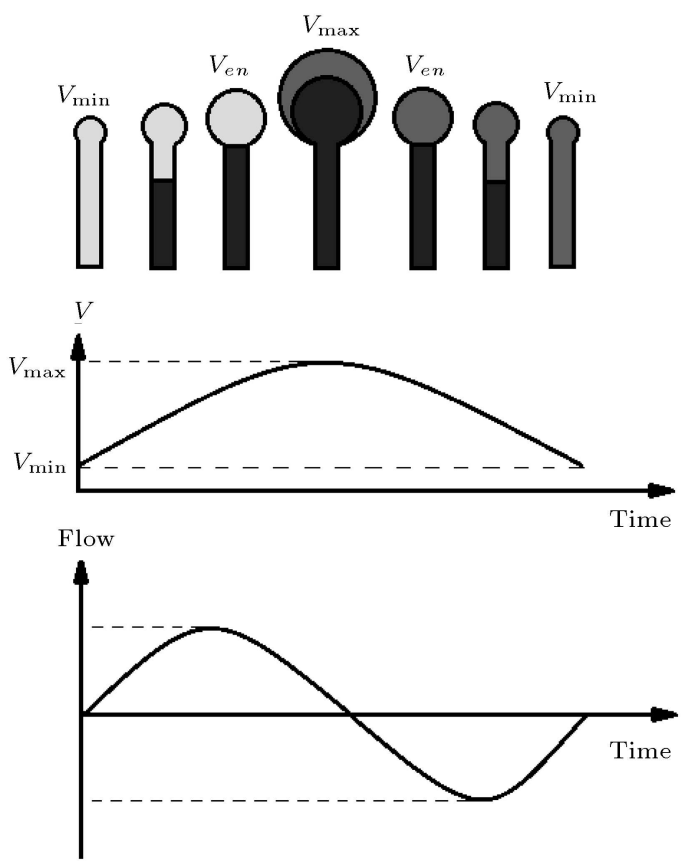

Figure 2. Schematic view of the variation of an alveolus volume during inhalation and exhalation. 
on impaction and diffusion. In the alveoli, due to the small velocity of the airflow and, in turn, particles, the impaction is insignificant and the deposition is calculated based on diffusion and sedimentation.

\subsection{Nasal and oral cavities deposition}

To calculate the deposition fraction in the oral and nasal cavities, mainly, the two mechanisms of impaction and diffusion are considered. Researchers have suggested different relations for the deposition fraction due to each of these mechanisms during the inhalation and exhalation. This study has tried to apply the most recent and reliable relations to calculate the deposition fraction in each part of the respiratory system.

\subsubsection{Nasal deposition during inhalation}

To calculate the deposition fraction due to impaction in the nasal cavity during the inhalation, the results of the research by Shang et al. were used [18]. They investigated the regional deposition of micro-particles in the nasal cavity and derived a diagram for the impaction deposition versus the inertial parameter. The inertial parameter is defined as $I=d^{2} Q$, where $d$ is the particle aerodynamic diameter and $Q$ is the volumetric airflow rate. Their research was published in 2015 and is one of the most recent researches in its field.

To calculate the deposition fraction in the nasal cavity due to diffusion, Eq. (3) is used as suggested by Shi et al. [19]. This relation is based on the diffusion parameter and Schmidt number.

$$
\eta=(0.568-0.69 \ln \Delta) \Delta^{1 / 2} \mathrm{Sc}^{-1 / 6} .
$$

Schmidt number in Eq. (3) represents the ratio of the momentum diffusivity in the fluid to the mass diffusivity of the particles. The diffusion parameter and Schmidt number are defined as $\Delta=\pi \bar{D} L /(4 Q)$ and Sc $=\nu / \bar{D}$, respectively. $\bar{D}, L, Q, \nu$ are the diffusion coefficient, the airway length, the airflow rate, and the kinetic viscosity of air, respectively.

\subsubsection{Nasal deposition during exhalation}

Compared to inhalation, little research has been done on oral and nasal deposition during the exhalation. The most recent and reliable relations for the nasal deposition fraction during the exhalation were presented by NCRP [20] in 1997. These relations that are presented in Eqs. (4) and (5) predict the particle deposition fraction in the nasal cavity during the exhalation due to impaction and diffusion, respectively. The subscript $p$ represents the particles' properties.

$$
\begin{aligned}
& \eta_{\text {imp }}=\left(1+\left(\rho_{p} d_{p}^{2} Q / 2300\right)^{1.01}\right)^{-1} \\
& \eta_{\text {diff }}=1-\exp \left(-15 \bar{D}^{1 / 2} Q^{-1 / 8}\right)
\end{aligned}
$$

These relations have been used for the exhalation particle deposition.

\subsubsection{Oral deposition during inhalation}

Golshahi et al. [21] in 2013 presented a new relation for the oral deposition fraction during the inhalation. In their experimental research, they made several replicas of the extrathoracic airways of a few adults and, by releasing micro-particles at the flow inlet, they measured the deposition fraction. Since their particle sizes were greater than $0.5 \mu \mathrm{m}$, this study considered their results as the impaction deposition. Their results represent the particle deposition fraction as a function of Reynolds number and Stokes number. This study used their proposed relation as follows:

$$
\eta=1-1 /\left[(1.5 e 5)\left(\operatorname{Stk}^{3.03} \operatorname{Re}^{0.25}\right)+1\right] .
$$

Reynolds and Stokes numbers are defined as $\mathrm{Re}=$ $4 \rho Q /(\pi \mu D)$ and Stk $=2 \rho_{p} d_{p}^{2} Q C_{c} /\left(9 \mu \pi D^{3}\right)$, respectively, where $\rho_{p}$ and $d_{p}$ are particle density and diameter, respectively, $Q$ is volumetric flow rate, and $D$ is the airway diameter.

To calculate $C_{c}$, Cunningham slip correction factor, one may refer to [22].

$\mathrm{Xi}$ and Longest [23] suggested a relation that predicted the oral deposition due to diffusion during the inhalation. Their research has been conducted recently and found to be more complete than the other related researches. It contains several geometric parameters of the oral cavity; for example, the total surface area and the hydraulic diameter of the inlet section are en gaged. In this relation, similar to its counterpart for the nasal cavity, the deposition fraction is a function of Reynolds and Schmidt dimensionless numbers. This relation is presented in Eq. (7):

$$
\eta=1-\exp \left(-0.1654 \frac{A_{\text {sur }}}{A_{\text {en }}} \operatorname{Re}^{-0.339} \mathrm{Sc}^{-0.661}\right) .
$$

In the above relation, the Reynolds number is calculated based on the hydraulic diameter of the inlet and is defined as $\operatorname{Re}=U_{e n} D_{h} / v$. The subscripts en and $h$ represent "entrance" and "hydraulic", respectively.

\subsubsection{Oral deposition during exhalation}

$\mathrm{Yu}$ et al. [24] predicted that the impaction deposition in the oral cavity during the exhalation was negligible, considering the oral cavity geometry and the flow pattern. Therefore, this study neglected the oral deposition during the exhalation due to impaction. To calculate the oral deposition during the exhalation due to diffusion, the relation based on NCRP [20] is used as follows:

$$
\eta=1-\exp \left(-8.51 \bar{D}^{1 / 2} Q^{-1 / 8}\right) .
$$

2.4.5. Total deposition in the oral and nasal cavities Considering the fact that both impaction and diffusion 
mechanisms take part in the deposition in the oral and nasal cavities, the addition law of probability is used to obtain the overall deposition fraction as follows:

$$
\eta_{t o t}=\eta_{d}+\eta_{i}-\eta_{d} \eta_{i}
$$

\subsection{Deposition in the tracheobronchial airways}

Different researchers have studied the deposition in the tracheobronchial airways. Yeh and Schum [1] proposed the relations based on the experimental data for the deposition fraction in the tracheobronchial airways. These relations were used by Koblinger and Hofmann [2] and Salma et al. [25,26]. The most comprehensive relation set to calculate the deposition fraction was suggested by NCRP [20] in 1997. However, Zhang et al. [27] presented even newer relations in 2008. These new relations are limited to a certain range of some parameters such as the particle size. Therefore, the present study used the newer relations in their own range of parameters; otherwise, the NCRP relations [20] were used. In the tracheobronchial airways, three mechanisms of impaction, sedimentation, and diffusion were considered to take part in the deposition.

\subsubsection{Deposition due to diffusion}

The diffusion phenomenon is a result of the wellknown Brownian motion of the particles. The diffusion coefficient represents the diffusivity of the particles. The deposition fraction of the particles in a typical volume is a fraction of the volume dimensions and the residence time of the particles, in addition to the particle's diffusivity. The relation suggested by Zhang et al. [27], which is a function of the diffusion parameter, is as follows:

$$
p_{d}=\Delta^{a} \exp (b-c \Delta) \text {. }
$$

The diffusion parameter in Eq. (10) is defined in Section 2.4.1, except that the length and airflow of the specific airway are used in the formula. In Eq. (10), $a$, $b$, and $c$ are constants that are determined based on the value of the diffusion parameter. For details, refer to Zhang et al. [27]. To calculate the deposition fraction due to diffusion in the tracheobronchial airways, by default, Eq. (10) is used unless its limitations are not authenticated. In such situations, the NCRP [20] relation is used. This relation is represented in Eq. (11).

$$
\begin{aligned}
p_{d}=1 & -0.819 e^{-14.63 \Delta}-0.0976 e^{-89.22 \Delta} \\
& -0.0325 e^{-228 \Delta}-0.0509 e^{-125.9 \Delta^{2 / 3}} .
\end{aligned}
$$

In the pause time, the deposition fraction is calculated by Eq. (12), which has also been suggested by NCRP [20].

$$
p_{d}=1-\exp \left(-23.136 \frac{\bar{D} t_{\text {rest }}}{D^{2}}\right) \text {. }
$$

\subsubsection{Deposition due to impaction}

The impaction deposition is a result of the deviation of the particles from their initial streamline due to the curvature of the flow pattern. Larger and heavier particles and sharper curvature of the flow path result in more deviation and, thus, more deposition fraction due to impaction. Eq. (13) that is presented by NCRP [20] shows the deposition fraction due to impaction as a function of Stokes number and the angle of bifurcation:

$$
p_{i}=1-(2 / \pi) \cos ^{-1}(\theta \text { Stk })+(1 / \pi) \sin \left[2 \cos ^{-1}(\theta \text { Stk })\right] \text {. }
$$

\subsubsection{Deposition due to sedimentation}

The deposition due to sedimentation is a result of gravity. NCRP presented the relation in Eq. (14) for this part of deposition. This equation is a function of the settling velocity, flow velocity, the dimensions of the airway, and the gravity angle.

$$
p_{s}=1-\exp \left(-\frac{4}{\pi} \frac{v_{\mathrm{set}}}{U} \frac{L}{D} \cos (\omega)\right) .
$$

In Eq. (14), $\omega$ is calculated as $\omega=(\pi / 2)-\varphi$, in which $\varphi$ is the angle between the axis of the channel and the direction of the gravity field. The settling velocity that is a function of the particle properties, the gravity acceleration, the viscosity of the fluid, and Cunningham slip correction factor is calculated as follows:

$$
v_{\text {set }}=C_{c} \rho_{p} g d_{p}^{2} /(18 \mu) .
$$

Considering that the sedimentation deposition occurs because of gravity and is not dependent on the airflow, it should be calculated during the pause time, too. In Eq. (14), the $L / U$ ratio represents the residence time of the particles in the airway. Therefore, it can be replaced by $t_{\text {pause }}$ and used to calculate the deposition during the respiratory pause time. The result is presented in Eq. (15):

$$
p_{s}=1-\exp \left(-\frac{4}{\pi} \frac{v_{\text {set }}}{D} t_{\text {pause }} \cos (\omega)\right) .
$$

2.5.4. Total deposition in the tracheobronchial airways Koblinger and Hofmann [2] used a simple summation of the deposition fractions due to different mechanisms to obtain the total deposition fraction in each airway. However, since the three mechanisms are not independent, Goo and Kim [28] proposed the addition law of probability to calculate the total deposition fraction due to all the mechanisms, as presented in Eq. (16).

$$
p_{\text {tot }}=p_{d}+p_{i}+p_{s}-p_{d} p_{i}-p_{d} p_{s}-p_{i} p_{s}+p_{d} p_{i} p_{s} .
$$

In this study, the total deposition in each airway is calculated based on Eq. (16). 


\subsection{Particles deposition in the alveolar ducts} As mentioned in the geometry section, the geometric data based on Weibel et al. [13] is used to simulate the structure of the alveolar ducts. The other parameter effective in the alveolar deposition is the mixing factor. The mixing of the "new" air and the "old" air in the alveoli is responsible for the effect of the previous respiratory cycles on the next cycles. The mixing phenomenon is basically dependent on the respiration conditions and the particle size; however, Koblinger and Hofmann [2] suggested a constant value of 0.25 for the mixing factor. They presented this value based on the best fitting of their simulation results with the experimental data. The method for the application of the mixing factor comes below in detail. After the partial deposition of the aerosol mass entering the alveoli, 0.25 fraction of the undeposited particle mass is mixed with the air that exists initially in the alveoli before the beginning of the inhalation; the rest totally exits the alveoli during the exhalation. The mass fraction that is mixed with the old air exits the alveoli gradually until the exhalation ends. The alveolar deposition is calculated based on proper relations, and the remaining mass fraction in each alveolus is saved for calculating the exhalation. When the exhalation begins, the unmixed part of the particles mass, after calculating its deposition, exits the alveoli just like its entrance, and, in the following, the mixed part exits the alveoli gradually until the exhalation ends. The unmixed part is tracked in the respiratory tract, and its deposition during the exhalation is calculated; finally, its residual fraction totally exits the respiratory system. Since the exiting of the mixed part of the particles lasts until the end of the exhalation, a typical particle of this mass fraction may stop at any point of the respiratory tract at the end of the exhalation. For this part, the deposition fraction of the mass fraction is calculated at the stopping point of the particles, and the remaining part of this mass fraction is saved at its geometric position for the calculation of the next respiratory cycle.

For the alveolar regions, the deposition fraction is calculated in two parts: The first one is the alveolar part of the duct and the second one is the cylindrical part of it. The total deposition in an alveolar airway is obtained through a summation of these two deposition fractions. For this purpose, the presented relations by Koblinger and Hofmann [2] are used.

\section{Results and discussion}

In this study, the particle deposition in different regions of the respiratory system in each of the five lobes and for different particle sizes is calculated. For each lobe, a symmetric pathway is considered based on the lobespecific geometrical data, and the calculations are done

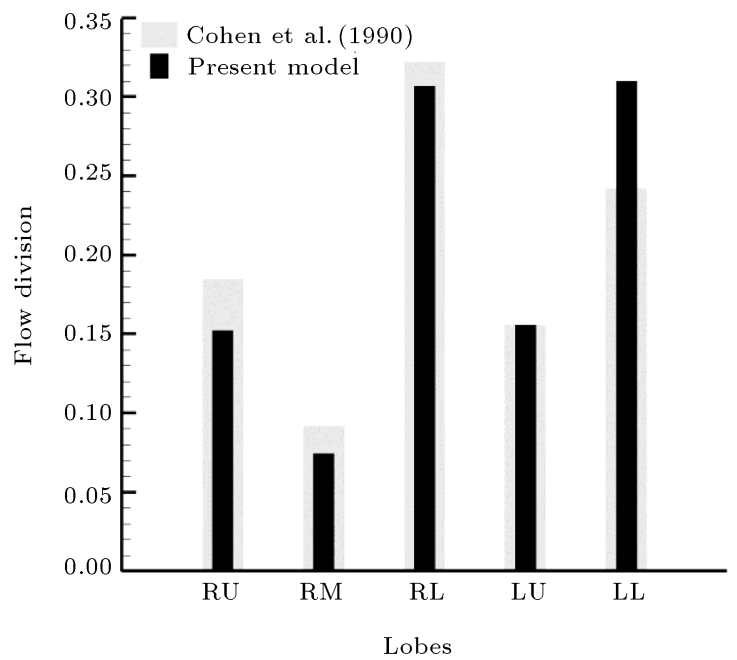

Figure 3. Flow division between the lobes.

for several successive respiratory cycles. The airflow in each airway is determined based on the alveoli number distal to it. Therefore, the share of each lobe from the total airflow is considered proportional to the number of the lobe alveoli. Figure 3 shows the share of each lobe from the total airflow. It can be observed that the left lower lobe and the right lower lobe draw the most share, and the right middle lobe receives the least share of flow. In Figure 3, the obtained flow division is also compared to the results of Cohen et al. [29]. As is shown, the trend is the same, but some differences can be detected. Cohen et al. [29] used the pressure outlet boundary condition to calculate the flow division. Therefore, the observed difference results from different methods. Since the main reason for the airflow is expansion and suction of the alveoli, it is more reasonable to calculate the airflow based on the alveoli number, which is pursued in the present study.

To calculate the deposition of each particle group in each airway, the flow of the airway at that specific moment is used. This technique generates more accurate computations than most of the previous studies that used the average flow rate in the calculations. The density of the particles is considered to be a standard value of $1 \mathrm{~g} / \mathrm{ml}$. Calculations are done for 37 different particle sizes ranging from $1 \mathrm{~nm}$ to $10 \mu \mathrm{m}$. The total respiratory deposition of different particle sizes is shown in Figure 4.

When breathing begins, theoretically, a few breathing cycles are required for the total deposition per cycle to reach a quasi-steady amount. Based on the particle size and the tidal volume, the number of cycles to reach a quasi-steady state can be different. To examine the validity of the results, the total calculated deposition in a single cycle, for a tidal volume of $500 \mathrm{ml}$, is compared to the other researchers' results. This comparison shown in Figure 4 shows to be in proper agreement with other results found by Koblinger and 


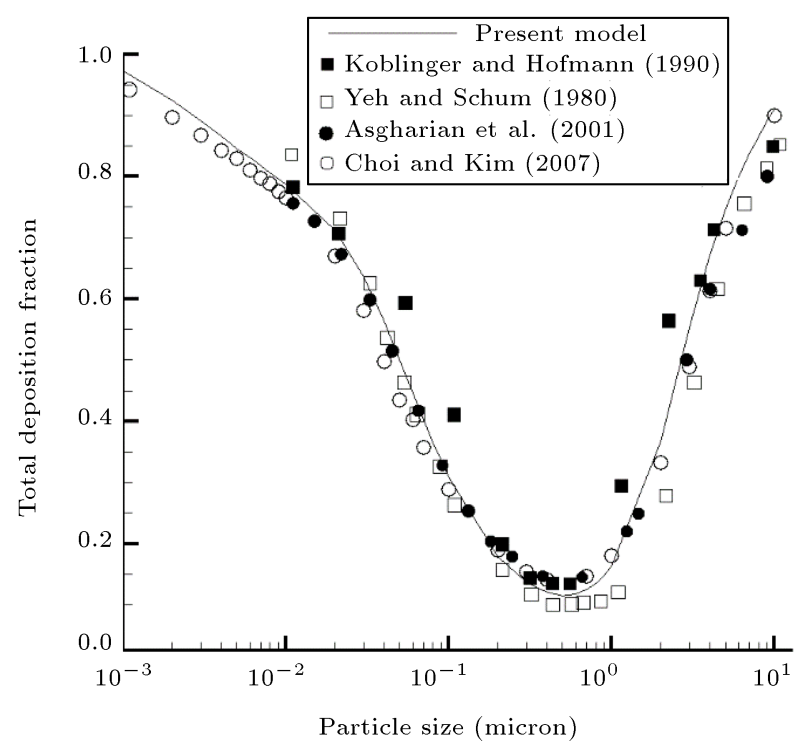

Figure 4. Total deposition fraction versus particle size for the $500 \mathrm{ml}$ tidal volume.

Hofmann [2], Yeh and Schum [1], Asgharian et al. [30], and Choi and Kim [31].

The total deposition fraction during a single cycle is compared with the total deposition fraction per cycle in the quasi-steady state. Figure 5 shows this comparison for tidal volumes of 500, 1000, and $1500 \mathrm{ml}$. The variation of the total deposition fraction less than $1 \%$ is considered as the criterion for reaching a quasisteady state.

As shown in Figure 5, for a particle size near $10 \mu \mathrm{m}$ and $1 \mathrm{~nm}$, the total respiratory deposition is

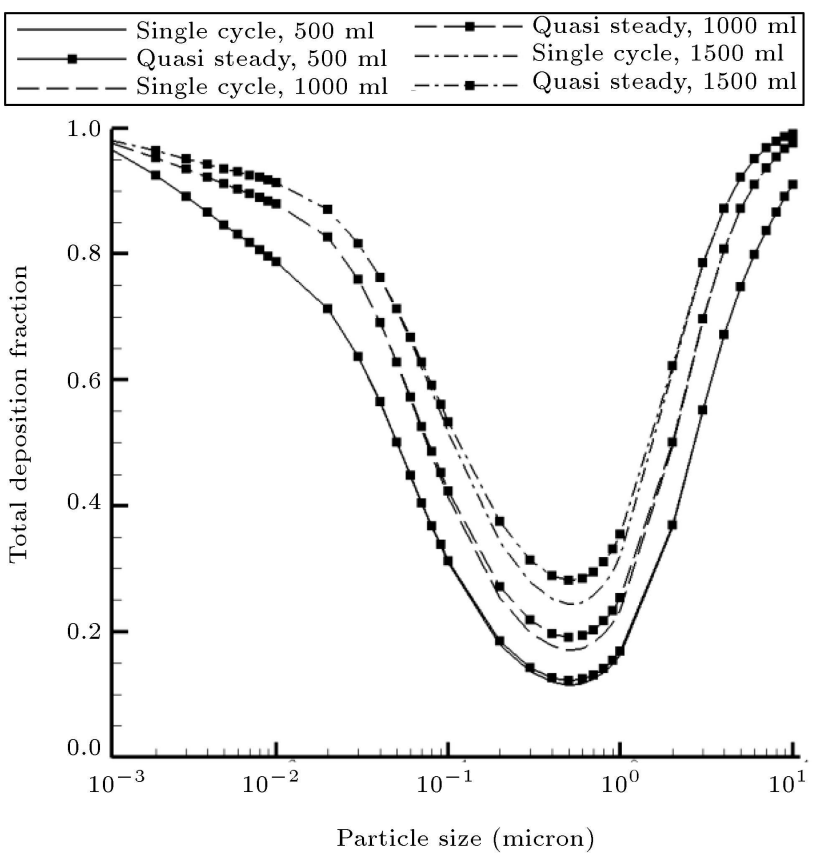

Figure 5. Total deposition per cycle for the different tidal volumes. close to unity. It means that these particles are almost totally deposited in the respiratory tract. The high deposition fraction of the particles near $10 \mu \mathrm{m}$ is due to their huge inertia that increases the impaction and sedimentation deposition in the airways. For a small particle near $1 \mathrm{~nm}$, which is quite small in size, the diffusion coefficient is so large that it causes high diffusive deposition in the respiratory tract. For the particles between these two limit sizes, the total deposition fraction decreases until it reaches its minimum value for the particle size about 0.5 to $0.6 \mu \mathrm{m}$. The particles in this size range are not massive enough nor small enough to be deposited totally in the respiratory tract. Therefore, there is a minimum for the total deposition fraction.

According to Figure 5, by increasing the tidal volume, the total deposition increases for all particle sizes. It can be observed that, for tidal volumes of 500,1000, and $1500 \mathrm{ml}$, the minimum deposition fractions are about $0.1,0.2$, and 0.25 , respectively. This increment results from an increase in the flow velocity in the airways and more penetration of the particles into the depth of the respiratory system. Moreover, it is obvious that by increasing the tidal volume, the difference between total deposition in a single cycle and the cycle that has reached the quasi-steady state increases. By increasing the tidal volume, the air entering the alveoli increases and more particles enter the alveoli. Consequently, more particle mass fraction is involved in the alveolar mixing. Considering that the alveolar mixing is the main reason for the difference between a single cycle and the cycle in the quasi-steady state, it is clear that this difference widens with an increase in the tidal volume, leading to the increment of the alveolar mixing.

The increment of deposition per cycle in the quasisteady state compared to a single cycle is shown in Figure 6 for three tidal volumes. The following facts can be inferred from the figure. For a tidal volume of $500 \mathrm{ml}$ that corresponds to the breathing at rest, in the quasi-steady state, the deposition increment is obvious for the particles between 0.2 and $1 \mu \mathrm{m}$; at the maximum, it reaches $5.7 \%$ for the $0.5 \mu \mathrm{m}$ particles. For tidal volumes of 1000 and $1500 \mathrm{ml}$, the maximum increment corresponds to $0.5 \mu \mathrm{m}$ particles and equals $12.4 \%$ and $15.5 \%$, respectively. Moreover, it can be inferred that by increasing the tidal volume from 500 to $1500 \mathrm{ml}$, the particle size range that shows the difference between the two mentioned states expands from 0.2 to $1 \mu \mathrm{m}$ corresponding to $500 \mathrm{ml}$ and from 0.07 to $2 \mu \mathrm{m}$ corresponding to $1500 \mathrm{ml}$. This occurs because as the tidal volume increases, the alveolar mixing becomes more important and more particle sizes are engaged.

To evaluate the difference between the results of geometric models, a comparison is made between the 


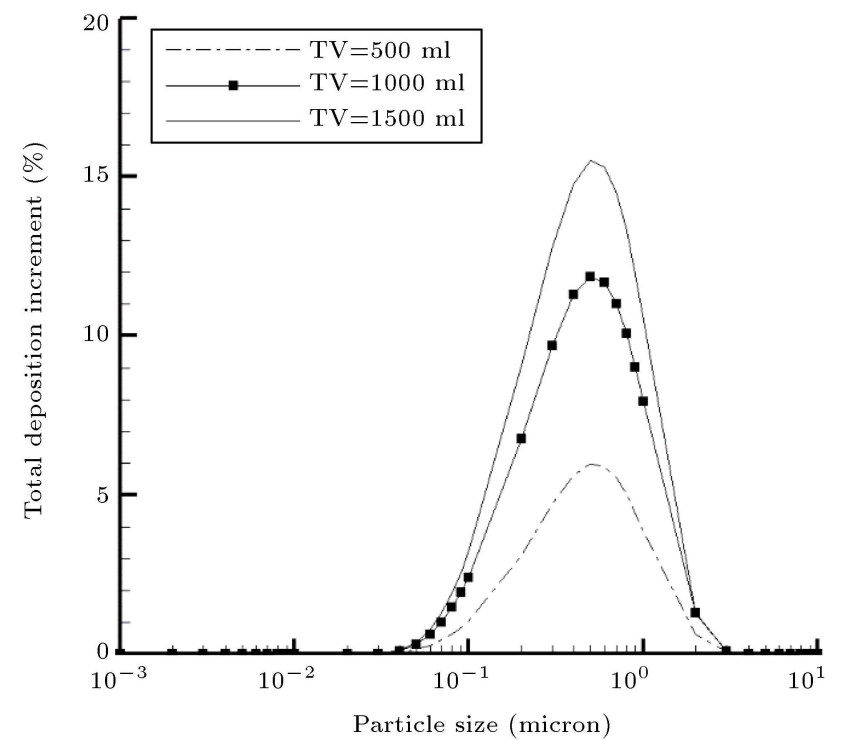

Figure 6. Deposition per cycle increment.

results of the present study-done based on a 5-lobe symmetric geometric model-and the results according to MPPD ${ }^{\complement} \mathrm{v} 2.1$ software. MPPD ${ }^{\complement} \mathrm{v} 2.1$, whose name is an acronym for "Multiple-Path Particle Dosimetry", was developed by Applied Research Associates, Inc. $(A R A)$ in 2009. This software has the capability to calculate the total, lobar, regional and generational particle deposition in the different geometric models in the respiratory system during a respiratory cycle. For the sake of our purpose, this program is run based on two geometric models: the proposed 5-lobe symmetric model and the stochastic geometric model. Figure 7 shows the results of total deposition in a single cycle for a tidal volume of $1000 \mathrm{ml}$, which is quite close to the daily outdoor physical activity state for the two models by MPPD and the present study based

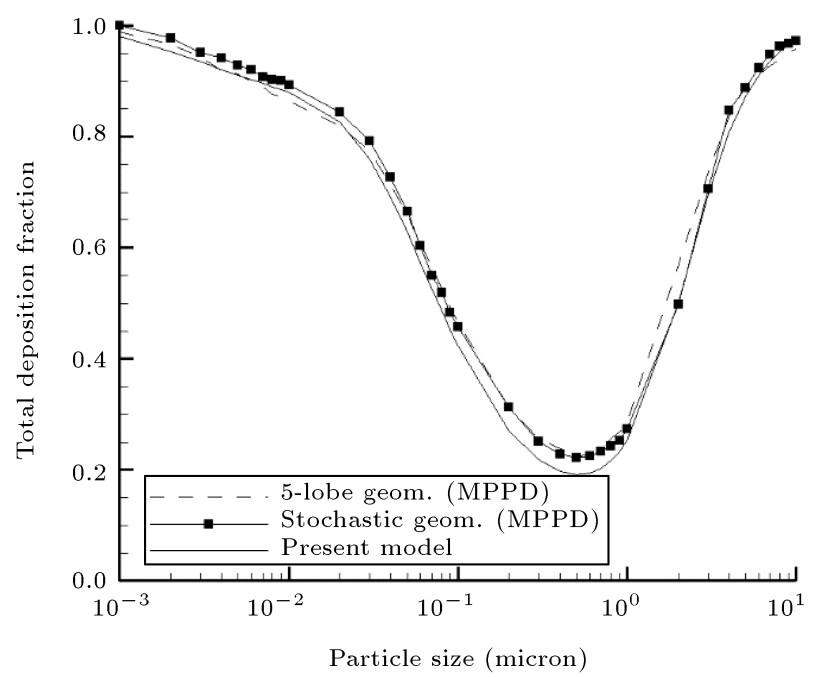

Figure 7. Total deposition fraction for the different geometric models. on the 5-lobe model. It can be observed that the results are quite in agreement. The difference in the total deposition between the present study and the stochastic model by MPPD reaches $16 \%$ at most and pertains to $0.6 \mu \mathrm{m}$ particles. On the other hand, the difference between the present study and the 5-lobe model by MPPD is of a lower percentage. Therefore, this investigation illustrates that a good estimate of the effect of successive respiratory cycles on the particle deposition can be achieved based on a 5-lobe symmetric model without any unacceptable deviation from the results of the more realistic stochastic geometric model.

Figure 8 shows a comparison of the deposition and the generation number between a single cycle and the cycle after that reaches a quasi-steady state for three tidal volumes and $0.5 \mu \mathrm{m}$ particles. It is obvious that the deposition per cycle in the quasisteady state is slightly more than that in a single cycle. This difference is more sensible in the case of the last generations, because the alveolar mixing happens in the last generations that are connected to the alveoli. Therefore, the effect of the successive cycles is more obvious in the last generations. In addition, it can be seen that the maximum deposition generation number increases with an increase in the tidal volume because of the greater penetration of particles into the respiratory tract. Besides, for a tidal volume of $500 \mathrm{ml}$, it can be observed that there is a little difference between a single cycle and the cycle in the quasi-steady state; by increasing the tidal volume, this difference widens due to the greater mass fraction of particles that reach the alveoli, where the alveolar mixing occurs.

Besides the mixing factor, the other parameter that has a role in the difference between the cycle in the quasi-steady state and a single cycle is the remaining

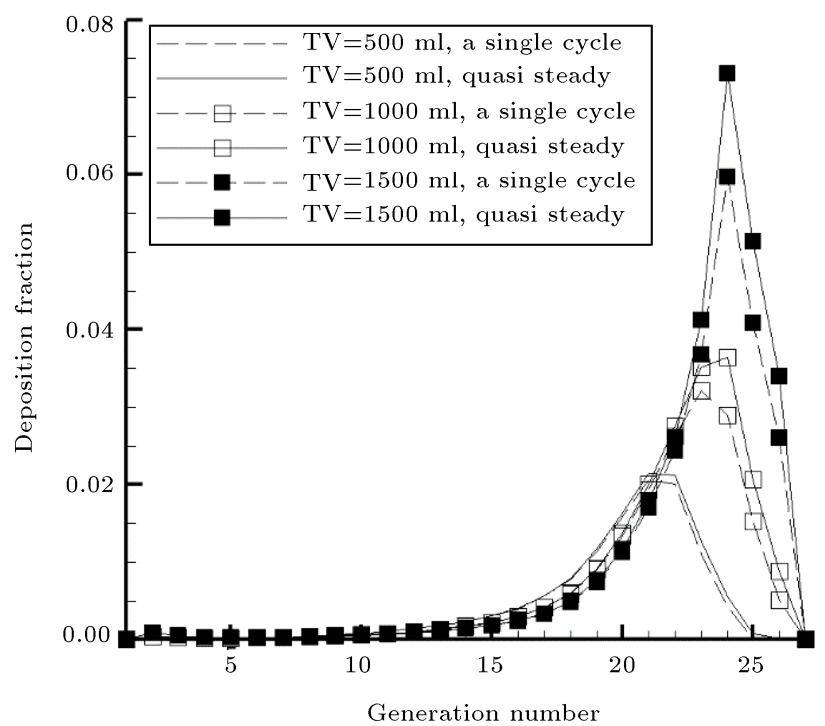

Figure 8. Deposition fraction versus generation number (0.5-micron particles). 


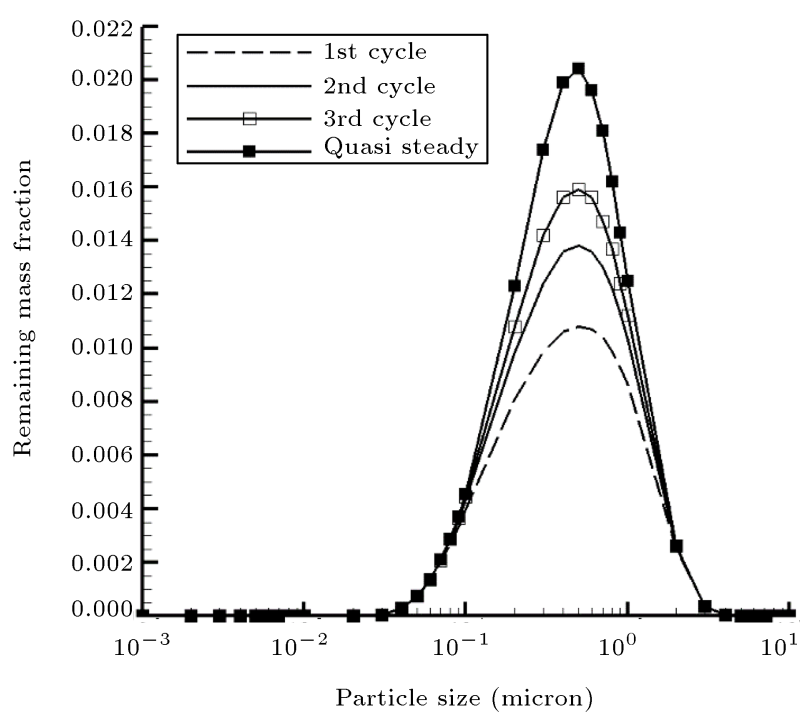

Figure 9. Remaining mass fraction at the end of cycles (500 $\mathrm{ml}$ of tidal volume).

mass fraction of particles in the respiratory tract at the end of each respiratory cycle. Considering the whole particles mass entering the respiratory system, part of it is deposited, part of it totally exits the respiratory system during the exhalation, and finally part of it remains suspended in the respiratory tract after the exhalation due to the delayed exit from the alveoli after the main wave of the particles, caused by the alveolar mixing. This remaining part increases the deposition per cycle in the next respiratory cycles. In the present study, as mentioned before, it is supposed that, at the beginning of the first respiratory cycle, the respiratory tract is totally empty of particles. At the end of the first cycle, some particle mass fraction remains in the respiratory tract. At the end of the second cycle, this remaining mass amount, somewhat increases. After a few cycles, in the quasi-steady state, the remaining mass fraction after each cycle reaches a constant value. Figure 9 shows the total remaining mass fraction in the respiratory system for a tidal volume of $500 \mathrm{ml}$ at the end of the successive respiratory cycles. It can be observed that, for this tidal volume, at the end of the first respiratory cycle, the maximum remaining mass fraction corresponds to $0.5 \mu \mathrm{m}$ particles and equals 0.011 fraction of the total entered particle mass. As is depicted in the figure, following the successive cycles that have reached the quasi-steady state, the aforementioned fraction value reaches about 0.022 of the total entered mass.

Variations in the tidal volume can also change the amount of the remaining mass fraction at the end of the cycles in the quasi-steady state. Figure 10 shows the total remaining mass fraction at the end of the cycles in the quasi-steady state for three values of the tidal volume. As is shown in the figure, the increment of the tidal volume can increase the remaining mass frac-

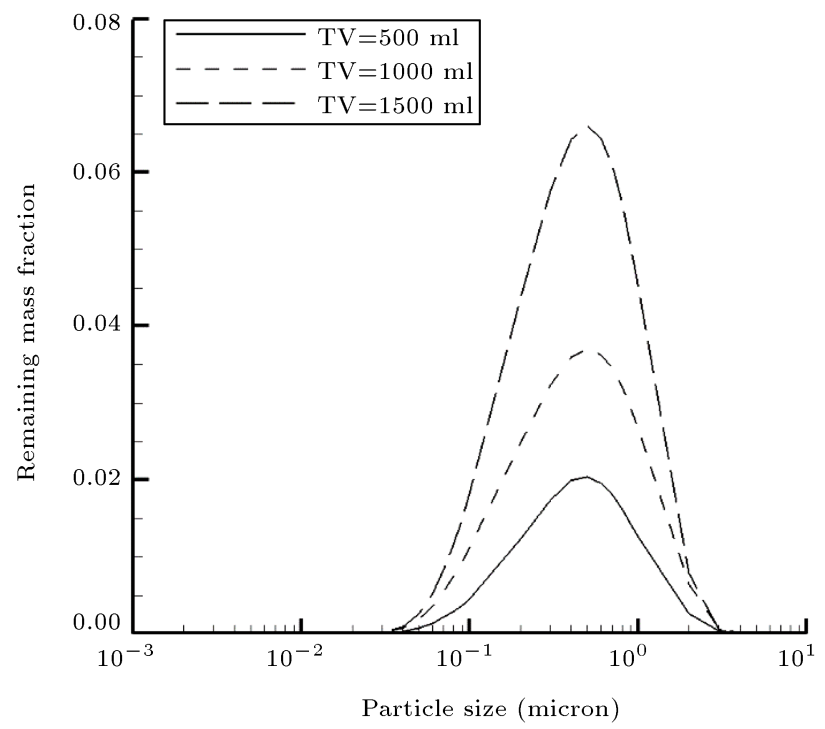

Figure 10. Remaining mass fraction in the quasi-steady state.

tion due to stronger alveolar mixing. The maximum remaining mass fraction that increases from 0.02 for $500 \mathrm{ml}$ tidal volume to 0.06 for $1500 \mathrm{ml}$ tidal volume corresponds to $0.5 \mu \mathrm{m}$ particles. It is shown that, for this particle size, none of the deposition mechanisms is strong enough to prevent the particles from reaching the alveolar region.

Besides the total remaining mass fraction, it is important to investigate the remaining mass fraction versus the generation number. Figure 11 shows the generational remaining mass fraction after the successive cycles for a tidal volume of $500 \mathrm{ml}$ and a particle size of $0.5 \mu \mathrm{m}$. It can be seen that, by passing the successive cycles and getting close to the quasisteady state, the generational remaining mass fractions

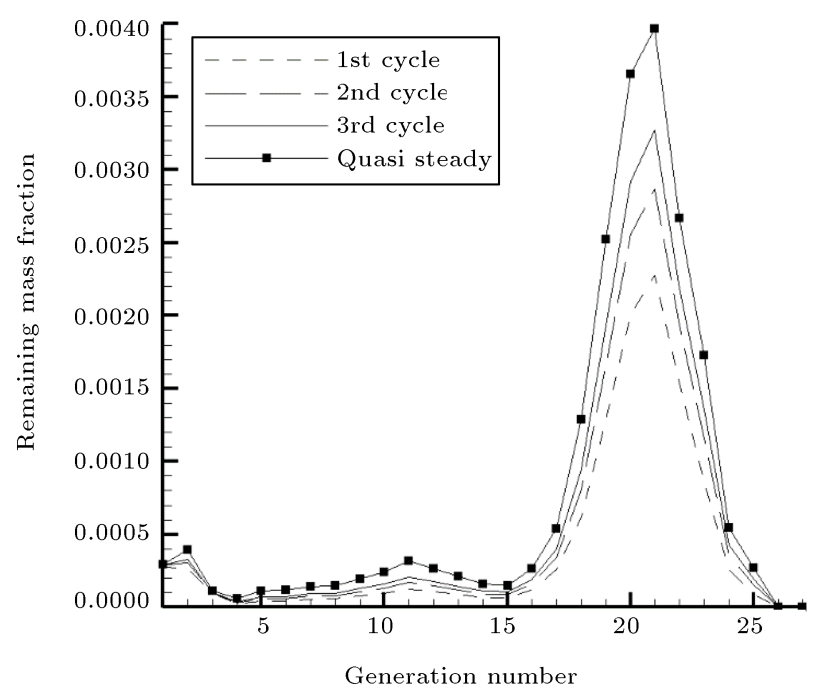

Figure 11. Generational remaining mass fraction at the end of cycles $(0.5 \mu \mathrm{m}$ particles, $\mathrm{TV}=500 \mathrm{ml})$. 
Table 1. The most required cycles to reach the quasi-steady state.

\begin{tabular}{ccc}
\hline $\begin{array}{c}\text { Tidal } \\
\text { volume } \\
(\mathbf{m l})\end{array}$ & $\begin{array}{c}\text { The most } \\
\text { needed } \\
\text { cycles }\end{array}$ & $\begin{array}{c}\text { Corresponding } \\
\text { particle size } \\
(\boldsymbol{\mu} \mathbf{m})\end{array}$ \\
\hline 500 & 2 & $0.4-0.9$ \\
1000 & 4 & 0.5 \\
1500 & 5 & $0.5-0.6$ \\
\hline
\end{tabular}

approach a certain profile. A maximum can be detected in this graph for the quasi-steady state. This maximum occurs at the 21st generation. As mentioned earlier, the alveolar mixing that is responsible for the difference between a single cycle and that cycle in the quasisteady state occurs in the last generations that are connected to the alveoli. Therefore, this is due to the strong effect of the alveolar mixing in this region. Of note, for the generational deposition fraction, the maximum appeared at the 21st generation, too.

Table 1 shows a large number of cycles required to reach the quasi-steady state and their corresponding particle sizes for three values of the tidal volume.

The criterion for reaching the quasi-steady state is considered when the total deposition fraction changes by less than $1 \%$ during a respiratory cycle. For medium-sized particles, a few respiratory cycles are required to reach the quasi-steady state. According to Table 1, by increasing the tidal volume, the number of needed cycles increases. This is due to the increment of the alveolar mixing. The highly required number of cycles corresponding to the particles around $0.5 \mu \mathrm{m}$ are 2,4 , and 5 cycles for the tidal volumes of 500,1000 , and $1500 \mathrm{ml}$, respectively.

According to Figure 10, it can be inferred that, for particles larger than $2 \mu \mathrm{m}$ and particles smaller than $0.05 \mu \mathrm{m}$, calculations can always be carried out based on a single cycle with great precision, because the effect of successive cycles is practically zero for these particles. This occurs because, for these particles, the deposition mechanisms are so strong that can prevent the particles from reaching the alveolar region and taking part in the alveolar mixing. Fortunately, most of the aerosolized medications, such as the inhalers and the nebulizers, mainly work in the particle size range of $1 \mu \mathrm{m}$ and larger. Therefore, for the calculations corresponding to these medical equipment pieces, we can safely use the results of a single cycle with enough precision and no excess computational cost is necessary.

To assess the sensitivity of the results to the amount of mixing fraction, total deposition fraction per cycle in the quasi-steady state is depicted in Figure 12 for different values of the mixing fraction. In this figure, total deposition fraction for the tidal volume of $1000 \mathrm{ml}$ is assessed with different values of mixing factors varying from 0 (no mixing) to 1 (perfect mixing). For this amount of the tidal volume, the

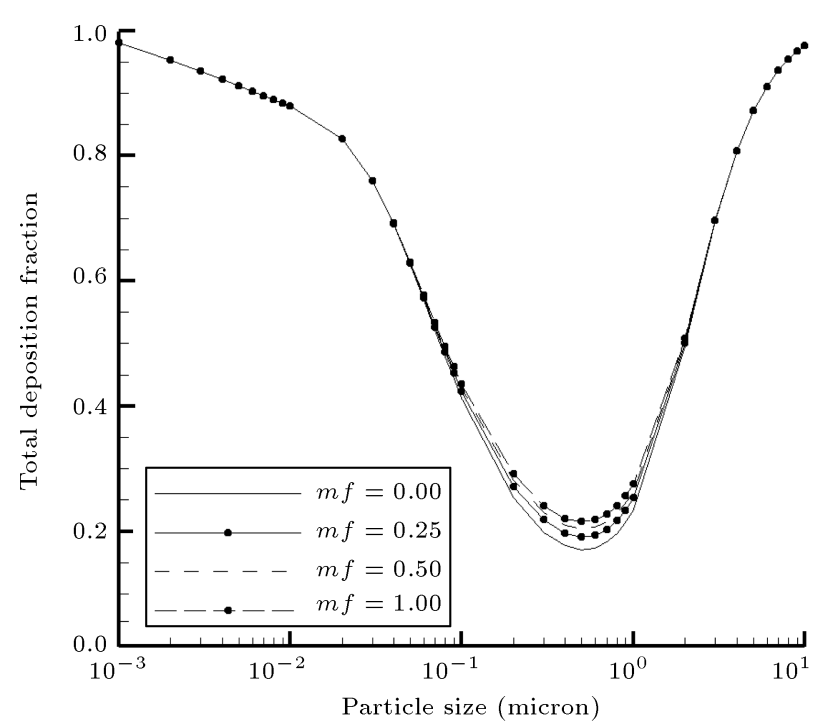

Figure 12. Total deposition per cycle for the different mixing factors and TV $=1000 \mathrm{ml}$.

variation of the mixing factor from 0.25 to 1 , at most, increases the deposition fraction by $12 \%$ and $25 \%$, respectively, pertaining to $0.5 \mu \mathrm{m}$ particles; further to that, for the other amounts of particle sizes, this increment is less. It is obvious that the amount of the mixing factor is not so effective such that a variation makes a significant difference in the deposition fraction of too large or too small particles. This is another verification of the claim that the effect of successive cycles is negligible for large and small particles and is partly sensible for the medium-sized particles.

\section{Conclusion}

In this study, the particle deposition in the respiratory system during the successive respiratory cycles was modeled. A 5-lobe symmetric geometric model was used, and the deposition formulas were applied according to different researchers to calculate the deposition fraction in each airway. Although the model did not fundamentally capture the physics of particle deposition process, its results were in good agreement with the experimental data. For three tidal volumes of 500, 1000, and $1500 \mathrm{ml}$ and for particle sizes ranging from $1 \mathrm{~nm}$ to $10 \mu \mathrm{m}$, the calculations were done and the results were extracted. The results of this work showed that, for different particle sizes and different values of tidal volume, a few cycles were required for deposition fraction to reach a quasi-steady state. Moreover, the comparison of the results of the present study for total deposition fraction and those of MPPD(C) v2.1 software showed that the difference between the proposed 5lobe model and the more realistic stochastic model was not unacceptable. This difference for the typical tidal volume of $1000 \mathrm{ml}$ turned out to be at most $16 \%$. Thus, in this study, a model was developed 
to make a good estimate of the effect of successive respiratory cycles on the total and generational particle deposition, though a 5-lobe symmetric model was used. The difference between the cycle in the quasi-steady state and a single respiratory cycle resulted from the alveolar mixing and the remaining mass fraction at the end of each respiratory cycle. The results showed that, for particles smaller than $0.05 \mu \mathrm{m}$ and particles larger than $2 \mu \mathrm{m}$, for all values of the tidal volumes, the remaining mass fraction was negligible due to the high deposition fraction, and the results of successive respiratory cycles were not different from those of a single cycle. For particles between 0.05 and $2 \mu \mathrm{m}$, due to the high penetration of the particles into the alveolar region, there was a difference between a single cycle and the cycle in the quasi-steady state. The maximum difference appeared at a particle size of $0.5 \mu \mathrm{m}$; as for this particle size, none of the deposition mechanisms was strong enough to avoid the particles to reach the alveolar region and became engaged in the alveolar mixing. For this particle size, the total deposition fraction in the cycle in the quasi-steady state was about 6,12 , and $16 \%$ greater than that in a single cycle for the tidal volume of 500, 1000, and $1500 \mathrm{ml}$, respectively. On the other hand, for low values of the tidal volume, due to the lower fraction of the particles reaching the alveolar region and weak alveolar mixing, the calculations of the cycle in the quasi-steady state were negligibly different from those of a single cycle; at high tidal volumes, this difference became significant. Therefore, keeping the aforementioned results in mind, for a wide range of applications, the results based on a single cycle can be used with reasonable accuracy to avoid unnecessary computational costs.

\section{References}

1. Yeh, H.-C. and Schum, G. "Models of human lung airways and their application to inhaled particle deposition", Bulletin of Mathematical Biology, 42, pp. 461-480 (1980).

2. Koblinger, L. and Hofmann, W. "Monte Carlo modeling of aerosol deposition in human lungs. Part I: Simulation of particle transport in a stochastic lung structure", Journal of Aerosol Science, 21, pp. 661674 (1990).

3. Hofmann, W. and Koblinger, L. "Monte Carlo modeling of aerosol deposition in human lungs. Part III: Comparison with experimental data", Journal of Aerosol Science, 23, pp. 51-63 (1992).

4. Koblinger, L. "Analysis of human lung morphometric data for stochastic aerosol deposition calculations", Physics in Medicine and Biology, 30, p. 541 (1985).

5. Koblinger, L. and Hofmann, W. "Aerosol deposition calculations with a stochastic lung model", Acta Physica Hungarica, 59, pp. 31-34 (1986).

6. Koblinger, L. and Hofmann, W. "Monte Carlo model for aerosol deposition in human lungs", Annals of Occupational Hygiene, 32, pp. 65-70 (1988).

7. Dastanpour, R., Monjezi, M., Saidi, M.S., and Pishevar, A. "Applying a realistic novel ventilation model based on spatial expansion of acini in a stochastic lung", Scientia Iranica, Transactions B, Mechanical Engineering, 21, p. 358 (2014).

8. Monjezi, M., Dastanpour, R., Saidi, M.S., and Pishevar, A. "Prediction of particle deposition in the respiratory track using 3D-1D modeling", Scientia Iranica, 19, pp. 1479-1486 (2012).

9. Georgakakou, S., Gourgoulianis, K., Daniil, Z., and Bontozoglou, V. "Prediction of particle deposition in the lungs based on simple modeling of alveolar mixing", Respiratory Physiology \& Neurobiology, 225, pp. 8-18 (2016).

10. Asgharian, B. and Price, O.T. "Deposition of ultrafine (nano) particles in the human lung", Inhalation Toxicology, 19, pp. 1045-1054 (2007).

11. Raabe, O., Yeh, H.-C., Schum, G., and Phalen, R.F., Tracheobronchial Geometry: Human, Dog, Rat, Hamster (1976).

12. Salma, I., Füri, P., Németh, Z., Balásházy, I., Hofmann, W., and Farkas, Á. "Lung burden and deposition distribution of inhaled atmospheric urban ultrafine particles as the first step in their health risk assessment", Atmospheric Environment, 104, pp. 3949 (2015).

13. Weibel, E.R., Sapoval, B., and Filoche, M. "Design of peripheral airways for efficient gas exchange", Respiratory Physiology \& Neurobiology, 148, pp. 3-21 (2005).

14. Ochs, M., Nyengaard, J.R., Jung, A., Knudsen, L., Voigt, M., Wahlers, T., Richter, J., and Gundersen, H.J.G. "The number of alveoli in the human lung", American Journal of Respiratory and Critical Care Medicine, 169, pp. 120-124 (2004).

15. Inthavong, K., Choi, L.-T., Tu, J., Ding, S., and Thien, F. "Micron particle deposition in a tracheobronchial airway model under different breathing conditions", Medical Engineering \& Physics, 32, pp. 1198-1212 (2010).

16. Tian, G., Longest, P.W., Su, G., Walenga, R.L., and Hindle, M. "Development of a stochastic individual path (SIP) model for predicting the tracheobronchial deposition of pharmaceutical aerosols: effects of transient inhalation and sampling the airways", Journal of Aerosol Science, 42, pp. 781-799 (2011).

17. Henry, F. and Tsuda, A. "Radial transport along the human acinar tree", Journal of Biomechanical Engineering, 132, p. 101001 (2010).

18. Shang, Y., Inthavong, K., and Tu, J. "Detailed microparticle deposition patterns in the human nasal cavity influenced by the breathing zone", Computers \& Flu$i d s$, 114, pp. 141-150 (2015).

19. Shi, H., Kleinstreuer, C., and Zhang, Z. "Dilute suspension flow with nanoparticle deposition in a representative nasal airway model", Physics of Fluids (1994-present), 20, p. 013301 (2008). 
20. N. C. o. R. Protection, Measurements. (National Council on Radiation Protection and Measurements) (1997).

21. Golshahi, L., Noga, M., Vehring, R., and Finlay, W. "An in vitro study on the deposition of micrometersized particles in the extrathoracic airways of adults during tidal oral breathing", Annals of Biomedical Engineering, 41, pp. 979-989 (2013).

22. Hinds, W.C. "Uniform particle motion", In Aerosol Technology: Properties, Behavior, and Measurement of Airborne Particles, 2nd Edn., pp. 48-51, WileyInterscience Pub., New York, USA (1982).

23. Xi, J. and Longest, P.W. "Effects of oral airway geometry characteristics on the diffusional deposition of inhaled nanoparticles", Journal of Biomechanical Engineering, 130, p. 011008 (2008).

24. Yu, C., Diu, C., and Soong, T. "Statistical analysis of aerosol deposition in nose and mouth", The American Industrial Hygiene Association Journal, 42, pp. 726733 (1981).

25. Salma, I., Balásházy, I., Hofmann, W., and Záray, G. "Effect of physical exertion on the deposition of urban aerosols in the human respiratory system", Journal of Aerosol Science, 33, pp. 983-997 (2002).

26. Salma, I., Balásházy, I., Winkler-Heil, R., Hofmann, W., and Záray, G. "Effect of particle mass size distribution on the deposition of aerosols in the human respiratory system", Journal of Aerosol Science, 33, pp. 119-132 (2002).
27. Zhang, Z., Kleinstreuer, C., and Kim, C.S. "Airflow and nanoparticle deposition in a 16-generation tracheobronchial airway model", Annals of Biomedical Engineering, 36, pp. 2095-2110 (2008).

28. Goo, J. and Kim, C.S. "Theoretical analysis of particle deposition in human lungs considering stochastic variations of airway morphology", Journal of Aerosol Science, 34, pp. 585-602 (2003).

29. Cohen, B.S., Sussman, R.G., and Lippmann, M. "Ultrafine particle deposition in a human tracheobronchial cast", Aerosol Science and Technology, 12, pp. 10821091 (1990).

30. Asgharian, B., Hofmann, W., and Bergmann, R. "Particle deposition in a multiple-path model of the human lung", Aerosol Science \& Technology, 34, pp. 332-339 (2001).

31. Choi, J.-I. and Kim, C.S. "Mathematical analysis of particle deposition in human lungs: an improved single path transport model", Inhalation Toxicology, 19, pp. 925-939 (2007).

\section{Appendix A}

\section{Flowcharts}

The flowcharts are presented in this section. Figure A.1. shows the deposition calculation flowchart in a tracheobronchial airway of a typical lobe, and Figure A.2. shows the deposition calculation flowchart

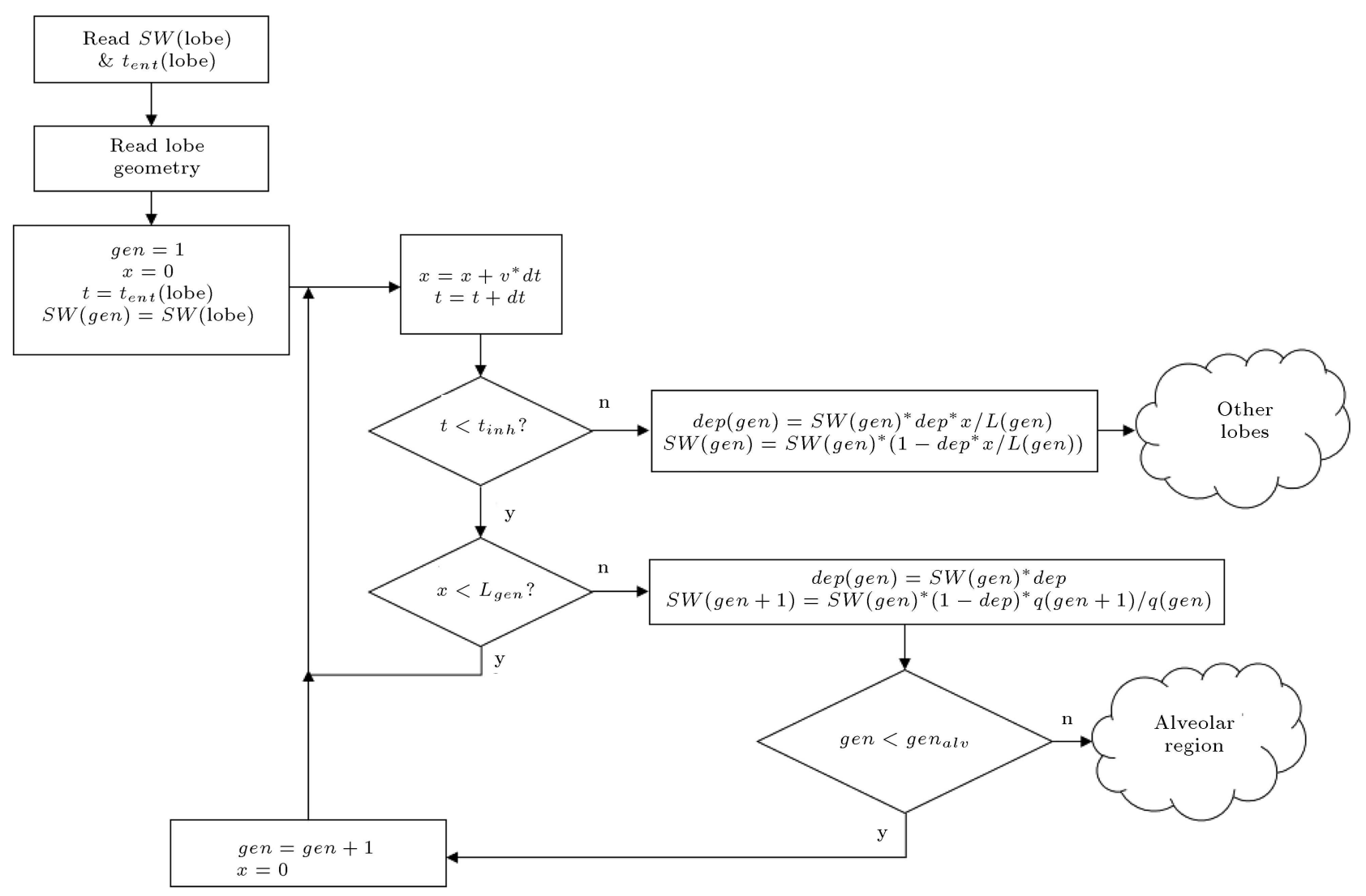

Figure A.1. Deposition calculation flowchart in a tracheobronchial airway of a typical lobe. 


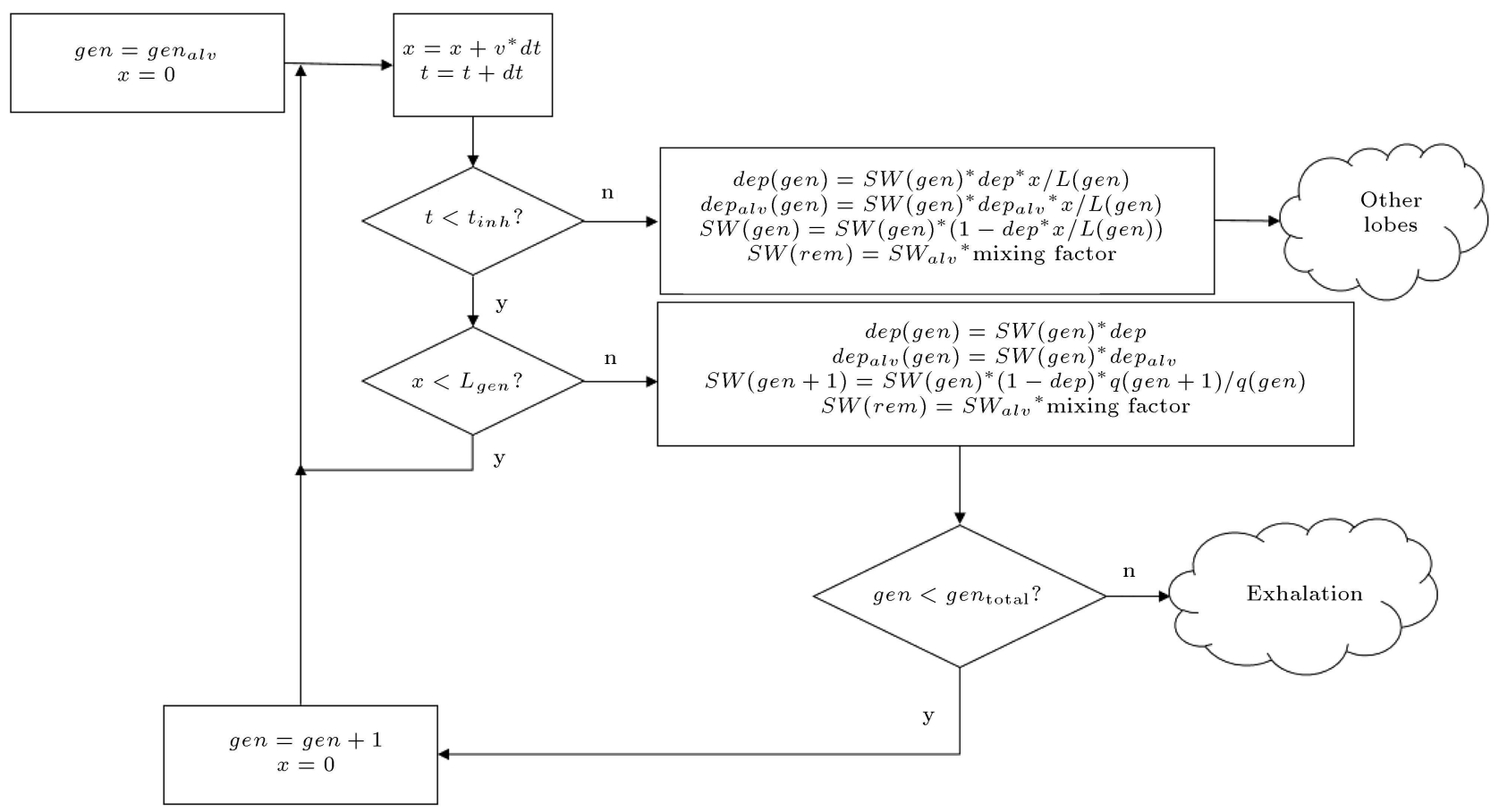

Figure A.2. Deposition calculation flowchart in an alveolar airway of a typical lobe.

in an alveolar airway of a typical lobe. Their details are presented in the main text.

\section{Biographies}

Hossein Nemati received his BSc degree in Mechanical Engineering specializing in Heat and Fluids from University of Isfahan, Isfahan, Iran in 2013 and MSc degree in Mechanical Engineering specializing in Energy Conversion from Sharif University of Technology, Tehran, Iran in 2016. His research interests are biological fluid mechanics, biological modeling, aerosol technology, and computational fluid dynamics.

Mohammad Said Saidi is a Professor of Mechanical Engineering at Sharif University of Technology, Tehran, Iran. His research interests include, but not limited to: CFD, micro and macro multiphase flows in human body, experimental design and mathematical modeling of microfluidic systems, and transport of nano and microscale aerosol particles. He earned his $\mathrm{PhD}$ in 1978 from M.I.T., USA.

Vahid Hosseini is an Associate Professor of Mechanical Engineering at Sharif University of technology. He is working in the field of combustion, internal combustion engine, and combustion generated nanoparticles and their health effects. He is also active in the field of air pollution studies ranging from atmospheric modeling to emission inventory and emission factor measurements. He earned his $\mathrm{PhD}$ from University of Alberta, Canada in 2008 and worked as a Researcher at the National Research Council of Canada before joining Sharif University. 